\title{
Surface and volume mode coupling experiments for high power mm-wave sources
}

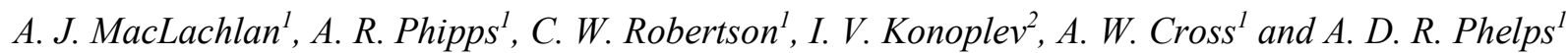 \\ ${ }^{I}$ Department of Physics, SUPA, University of Strathclyde, Glasgow, G4 ONG, Scotland, UK \\ ${ }^{2} \mathrm{JAI}$, Department of Physics, University of Oxford, Oxford, OX1 3RH, England, UK \\ a.d.r.phelps@strath.ac.uk
}

\begin{abstract}
Periodic surface lattice (PSL) structures have been fabricated and measured. When the required conditions are met, volume and surface waves can couple to form a cavity eigenmode at a frequency determined by the PSL's parameters. The formation of such eigenmodes is relevant to the realization of high-power mm-wave and $\mathrm{THz}$ coherent sources.
\end{abstract}

Keywords-periodic surface lattice; PSL; surface mode; volume mode; mode coupling; mm-wave sources.

\section{INTRODUCTION}

Research involving the exploitation of electromagnetic (EM) fields inside and on the surface of periodic lattices is relevant to the realization of high-power, coherent radiation sources operating in the $\mathrm{GHz}-\mathrm{THz}$ frequency regimes. Such sources are suited to various applications including imaging and security, communications, the quality control of pharmaceutical products and monitoring atmospheric dust clouds and space debris. In order to avoid multi-mode excitation, which is detrimental to the efficient operation of the device, the cavity dimensions of EM sources tend to be comparable to the intended operating wavelength of the source. This decrease in cavity size with wavelength restricts the power output capability of mm-wave and $\mathrm{THz}$ sources. Overcoming this challenge, by introducing novel methods of mode selection in oversized cavities, allows high power output capabilities to be maintained at mm-wave and $\mathrm{THz}$ frequencies [1-9]. The technique proposed in this work involves the use of periodic surface lattices (PSLs) which, under certain conditions, are shown to facilitate coupling of volume and surface fields, resulting in the formation of a single cavity eigenmode. These structures may be described as high-impedance surfaces or effective metamaterials due to their small corrugation depth in relation to their operating frequency. PSLs of planar geometry, used to demonstrate the fundamental "proof of principle" coupling of volume and surface fields, are considered in this work. Such PSLs can be fabricated into cylindrical structures which can be combined with a suitable electron beam via conformal mapping.

Planar PSLs with different periodicities (1.50mm, $1.62 \mathrm{~mm}, 1.74 \mathrm{~mm}$ and $1.94 \mathrm{~mm}$ ) designed to operate at the $140-220 \mathrm{GHz}$ frequency band, were obtained using laser and chemical etching techniques. The simplest structures, consisting of $0.3 \mathrm{~mm}$ thick, copper PSLs without substrates, designed to study the surface field exclusively, were fabricated using laser etching. These PSLs share some similarities to inductive mesh structures used as high bandpass filters [10]. Planar PSLs mounted on dielectric substrates, with a transverse size of 40 periods and corrugation depth of $35 \mu \mathrm{m}$, were fabricated by etching copper coated FR-4 sheets. To further vary the structures' properties, FR-4 substrates of three different thicknesses $(0.4 \mathrm{~mm}, 0.8 \mathrm{~mm}, 1.4 \mathrm{~mm})$ and permittivity $(5.69,4.71,4.45)$ were used. The permittivity of each sample was established by measuring the phase of a $140-220 \mathrm{GHz}$ signal launched through the sample. For some structures, the copper backing of the coated dielectric sheets was left intact, whilst in the others, the copper was entirely dissolved. It has been shown that the copper backing improves the synchronisation of the lattice by confining the volume field within the dielectric and facilitating the coupling of volume and surface fields.

The PSLs are scalable and can be tailored to operate over different frequency bands. A shorter wavelength, $0.63 \mathrm{~mm}$ PSL designed to operate within the $325-500 \mathrm{GHz}$ band is photographed in fig.1.

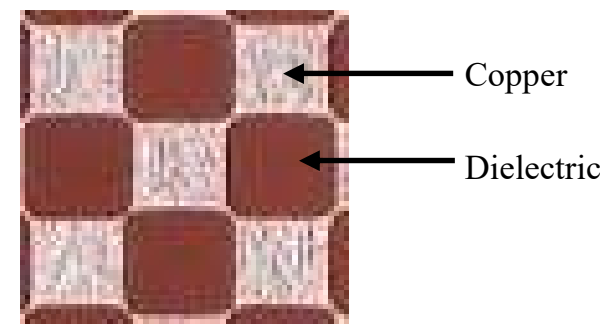

Fig. 1. Magnified image showing a section of the $0.63 \mathrm{~mm}$ PSL structure designed to operate at $325-500 \mathrm{GHz}$. The PSL was fabricated using chemical etching techniques and photographed using a Hirox microscope.

\section{RESULTS}

Experimental measurements were carried out using an Anritsu Vector Network Analyzer (VNA) complemented by a pair of high-frequency heads as shown in fig.2. The PSLs were measured over various incident angles $\theta_{i}$, whilst keeping the angles of the launching and receiving horns equal, $\theta_{i}=\theta_{r}$. For the set of mesh PSL structures without substrates, a sharp surface field resonance was measured at a frequency determined by the PSL's periodicity.

The surface field resonances were found to exhibit a clear angular dependence, shifting down in frequency with increasing angle.

With the inclusion of a dielectric substrate but no copper backing, multiple resonances were observed. Fig. 3 shows the reflected power for a $1.62 \mathrm{~mm}$ PSL etched onto a $0.4 \mathrm{~mm}$ FR-4 substrate and measured over an angular range of $\theta_{i}=$ $40^{\circ}-65^{\circ}$. Resonance 3 corresponds to the PSL's surface 
field, while $\mathbf{1}$ is an internally reflected volume mode. Resonance $\mathbf{2}$ is suggestive of weak eigenmode formation due to the poorly synchronised PSL.

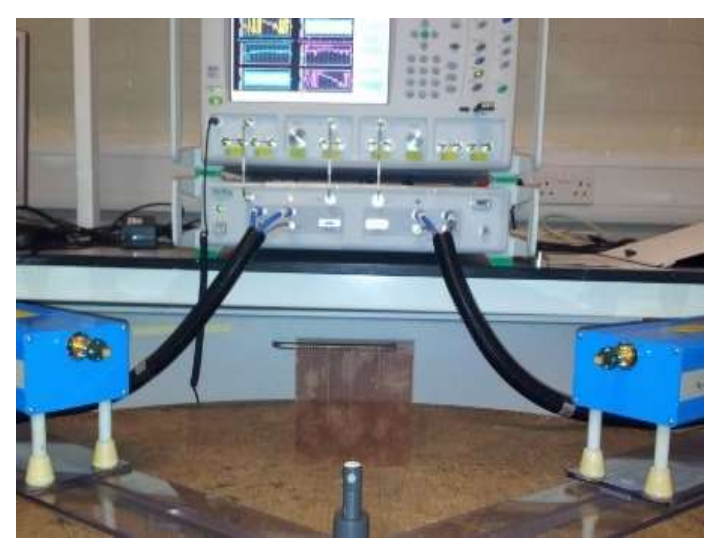

Fig. 2. Photograph showing measurements of the $1.50 \mathrm{~mm}$ mesh PSL made at various incident angles. A pair of G-band horns were connected to 140$220 \mathrm{GHz}$ heads of a Vector Network Analyzer (VNA) used to evaluate the scattering parameters.

The surface field resonances were found to exhibit a clear angular dependence, shifting down in frequency with increasing angle.

With the inclusion of a dielectric substrate but no copper backing, multiple resonances were observed. Fig.3 shows the reflected power for a $1.62 \mathrm{~mm}$ PSL etched onto a $0.4 \mathrm{~mm}$ FR-4 substrate and measured over an angular range of $\theta_{i}=$ $40^{\circ}-65^{\circ}$. Resonance 3 corresponds to the PSL's surface field, while $\mathbf{1}$ is an internally reflected volume mode. Resonance $\mathbf{2}$ is suggestive of weak eigenmode formation due to the poorly synchronised PSL.

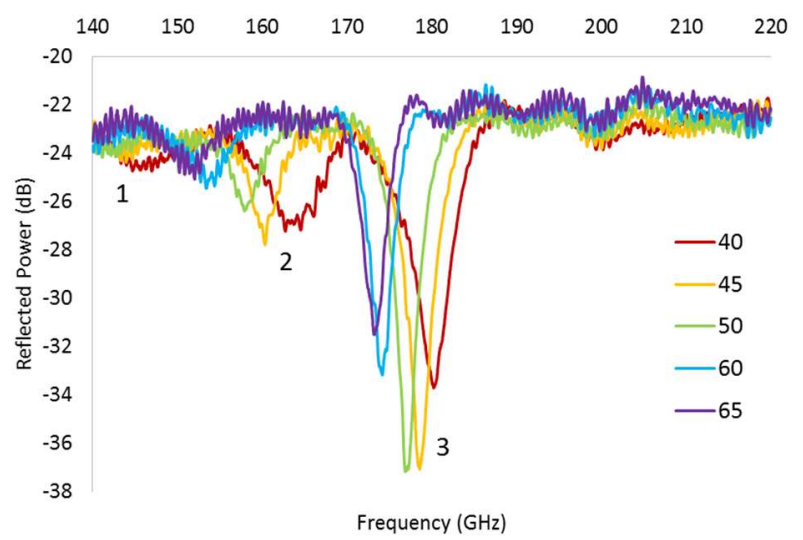

Fig. 3 Reflection measurement for a $1.62 \mathrm{~mm}$ PSL mounted on a $0.4 \mathrm{~mm}$ FR-4 substrate with no copper backing. Three resonances relating to volume and surface fields are observed.

Coherent eigenmode formation observed for a $1.62 \mathrm{~mm}$ PSL mounted on a $0.8 \mathrm{~mm}$ copper-backed substrate at a fixed incident angle of $45^{\circ}$ is shown in fig.4.

The three overlaid traces which were recorded on three different occasions separated in time by several months demonstrate the reproducibility of the results. When the copper is positioned behind the PSL and substrate in an arrangement similar to a Fabry-Perot cavity (with the PSL and copper backing acting as mirrors) the individual lattice perturbations are synchronised by the volume field inside the dielectric. The single resonance is 'mode-locked' to a particular frequency and no longer shifts with incident angle. Such high-Q cavities, when mapped into cylindrical geometry can form the basis of a novel coherent source when excited by a suitable electron beam passing through the cylindrical cavity. The advantage of using such PSLs to form eigenmodes in overmoded structures is that they facilitate the disruption of the usual scaling of output power with frequency. Instead of using conventional scaling ratios of $D / \lambda \sim 1$, where $D$ represents the diameter of the cylindrical structure and $\lambda$ is the wavelength, using this method of eigenmode formation and selection, it is believed to be realistic to achieve coherent, relatively efficient radiation sources with $\mathrm{D} / \lambda \sim 7$ and eventually $\mathrm{D} / \lambda \sim 10$. The latter represents a hundredfold increase in the crosssectional area of the interaction structure, with consequential increases in the maximum output power obtainable from short wavelength mm-wave and sub-THz sources. Because of the mode selectivity provided by the volume mode/surface mode coupling mechanism the device power output and efficiency are not expected to decrease, as is usually the case when insufficient mode selectivity is provided and an overmoded device operates in a multimode manner.

The results of this generic work suggest that sources working at several 100's of GHz can be designed to provide output powers and efficiencies comparable with conventional sources with $\mathrm{D} / \lambda \sim 1$ operating at lower frequencies such as $\sim 100 \mathrm{GHz}$.

It should be noted that although printed circuit boards (PCBs) involving dielectric materials have been used for these planar two dimensional "proof of principle" PSL experiments, in an electron beam driven cylindrical PSL the structure is intended to be all-metallic and fully compatible with high vacuum, high power electron beams [11,12] and intense electromagnetic fields. The coherent combination of electromagnetic fields within an over-sized/overmoded structure to produce higher powers is an alternative approach to coherently combining electromagnetic fields together externally from several phase-synchronised discrete sources [13].

The construction of all-metallic cylindrical PSL structures based on the same fundamental mode-coupling principles and suitable for being excited and driven by an electron beam is the subject of another related paper being presented in this Workshop [14].

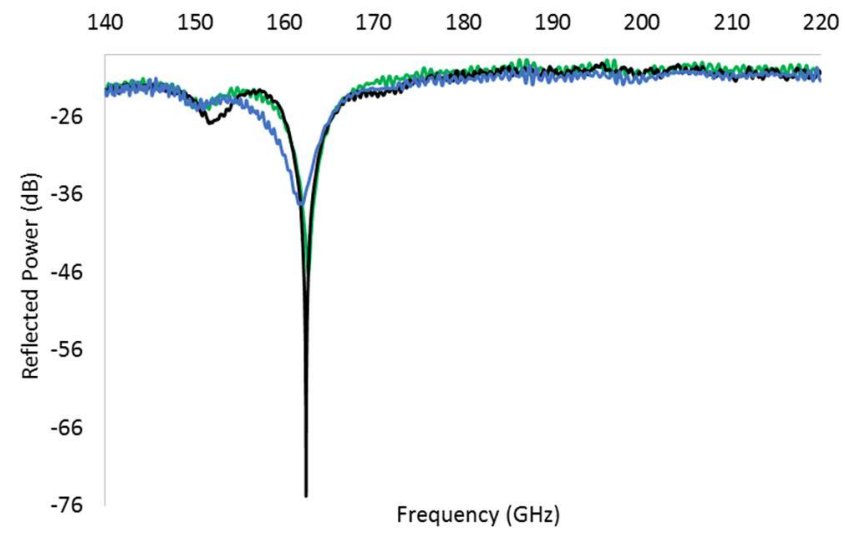

Fig. 4 Coherent eigenmode formation due to coupling of the surface and volume fields for a $1.62 \mathrm{~mm}$ PSL etched onto a $0.8 \mathrm{~mm}$ FR-4 substrate with a $35 \mu \mathrm{m}$ copper foil backing measured at $45^{\circ}$. The 3 overlaid traces demonstrate the reproducibility of the measurements. 


\section{SUMMARY}

The coupling of volume and surface modes and coherent eigenmode formation in planar PSL structures has been successfully demonstrated. The parameters of these structures were carefully chosen to facilitate coherent eigenmode formation and to obtain a single high $Q$ resonance suitable for use in a high-power, high frequency source.

When the necessary conditions are met, PSL structures have the potential to provide effective interaction regions for novel, coherent sources of radiation. All the PSLs considered are scalable and are therefore applicable to a broad range of frequencies from the microwave and $\mathrm{mm}$ wave range and stretching into the $\mathrm{THz}$ and far-infrared regions of the electromagnetic spectrum.

\section{ACKNOWLEDGMENT}

Amy J. MacLachlan and A. R. Phipps thank the EPSRC for supporting their postgraduate studentships. This work was partly funded by a Leverhulme Trust International Grant Network on "Advanced research on generation of $\mathrm{THz}$ and X-ray radiation". The authors would also like to thank EOARD for partial support of this work.

\section{REFERENCES}

[1]. N. S. Ginzburg, N. Y. Peskov, A. S. Sergeev, et al. , "Theory of freeelectron maser with two-dimensional feedback driven by an annular electron beam", J. Appl. Phys., vol. 92, pp. 1619-1629, Aug. 2002.

[2]. N.S. Ginzburg, N. Y. Peskov, A. S. Sergeev, et al., "The use of a hybrid resonator consisting of one dimensional and two-dimensional Bragg reflectors for generation of spatially coherent radiation in a co-axial freeelectron laser", Phys. Plasmas, vol. 9, pp. 2798-2802, June 2002.

[3]. I. V. Konoplev, A. W. Cross, A. D. R. Phelps, et al., "Experimental and theoretical studies of a coaxial free-electron maser based on twodimensional distributed feedback", Phys. Rev. E, vol. 76, 056406, Nov. 2007.

[4]. I. V. Konoplev, L. Fisher, A.W. Cross, et al., "Surface wave Cherenkov maser based on a periodic lattice", Appl. Phys. Lett., vol. 96, 261101, June 2010 .

[5]. I. V. Konoplev, L. Fisher, K. Ronald, et al., "Surface-field cavity based on a two dimensional cylindrical lattice," Appl. Phys. Lett., vol. 96, 231111, June 2010

[6]. A.W. Cross, I. V. Konoplev, A. D. R. Phelps and K. Ronald, "Studies of surface two-dimensional photonic band-gap structures", J. Appl. Phys., vol. 93, pp. 2208-2218, Feb. 2003.

[7]. I. V. Konoplev, A. J. MacLachlan, C. W. Robertson, A. W. Cross and A D. R. Phelps, "Cylindrical, periodic surface lattice - Theory, dispersion analysis and experiment”, Appl. Phys. Lett., vol. 101, 121111, Sept. 2012.

[8]. I. V. Konoplev, A. J. MacLachlan, C. W. Robertson, A. W. Cross and A D. R. Phelps, "Cylindrical periodic surface lattice as a metadielectric: concept of a surface-field Cherenkov source of coherent radiation", Phys. Rev. A, vol. 84, 013826, July 2011.

[9]. I. V. Konoplev, A. R. Phipps, A. D. R. Phelps, C. W. Robertson, K. Ronald and A. W. Cross, "Surface field excitation by an obliquely incident wave," Appl. Phys. Lett., vol. 102, 141106, April 2013.

[10]. R. Ulrich, "Far-Infrared Properties of Metallic Mesh and its Complementary Structure," Infrared Phys., vol. 7, pp.37-55, March 1976.

[11]. I. V. Konoplev, A. W. Cross, P. MacInnes, W. He, C. G. Whyte and A. D. R. Phelps, "High current oversized annular electron beam formation for high-power microwave research," Appl. Phys. Lett., vol. 89, 171503, Oct. 2006.

[12]. A. W. Cross, H. Yin, W. He, K. Ronald, A. D. R. Phelps and L. C. Pitchford, "Generation and application of pseudospark-sourced electron beams," J. Phys. D: Appl. Phys., vol. 40, pp. 1953-1956, March 2007.

[13]. N. S. Ginzburg, A. W. Cross, A. A. Golovanov, et al., "Generation of electromagnetic fields of extremely high intensity by coherent summation of Cherenkov superradiance pulses," Phys. Rev. Lett., vol. 115, 114802 , Sept. 2015.

[14]. A. R. Phipps, A. J. MacLachlan, C. W. Robertson, A. W. Cross, and A. D. R. Phelps, "Additive manufacturing method of prototyping novel mm-wave and THz sources," UCMMT2016, Proceedings of $9^{\text {th }}$ IEEE UKEurope- China Workshop on Millimetre Waves and THz Technologies, Qingdao, China, 5-7 Sept. 2016. 